\title{
A SCADA System for Energy Management in Intelligent Buildings
}

\author{
João Figueiredo*, José Sá da Costa • \\ * CEM/IDMEC, Universidade Évora, R. Romão Ramalho, 59; 7000-671 Évora, Portugal, \\ • IDMEC, Instituto Superior Tecnico, Technical University of Lisbon; 1049-001 Lisboa, Portugal
}

\begin{abstract}
This paper develops an energy management platform for intelligent buildings using a SCADA system (Supervisory Control And Data Acquisition). This SCADA system integrates different types of information coming from the several technologies present in modern buildings (control of ventilation, temperature, illumination, etc.).
\end{abstract}

The developed control strategy implements an hierarchical cascade controller where inner loops are performed by local PLCs (Programmable Logic Controller), and the outer loop is managed by a centralized SCADA system, which interacts with the entire local PLC network.

In this paper a Predictive Controller is implemented above the centralized SCADA platform. Tests applied to the control of temperature and luminosity in huge-area rooms are presented. The developed Predictive Controller optimizes the satisfaction of user explicit preferences coming from several distributed user-interfaces, subjected to the overall constraints of energy waste minimization.

In order to run the Predictive Controller with the SCADA platform a communication channel was developed to allow communication between the SCADA system and the MATLAB application where the Predictive Controller runs.

Index Terms - Energy Management, SCADA Systems, Building Automation, Predictive Control. 